\title{
Geschlechtsdifferenzen in der Zukunftsorientierung ${ }^{1}$
}

\author{
Gisela Trommsdorff \\ Institut für Erziehungswissenschaft, TH Aachen \\ Eilfschornsteinstraße 7, D-5100 Aachen
}

\author{
Christine Burger \\ Traudl Füchsle \\ Sonderforschungsbereich 24, Universität Mannheim (WH) \\ L 13, 15-17, D-6800 Mannheim
}

$\mathrm{Z}$ u s a m m e $\mathrm{n}$ f a s s u $\mathrm{ng}$ : Um den Einfluß von Geschlechtsrollen und Bildungsstand auf die Art und Ausprägung der $Z$ ukunftsorientierung $(\mathrm{ZO})$ empirisch zu erfassen, wurden 256 weibliche und männliche Jugendliche von Berufsschulen, Gymnasien und der Universität mit einem - durch Item- und Faktorenanalysen statistisch erprobten - Zukunftsorientierungs-Instrument befragt. Entgegen den einfachen Hypothesen und Befunden der Literatur, daß Frauen ihre Zukunft stärker in bezug auf den familialen Bereich, Männer dagegen eher in bezug auf den Berufsbereich hin strukturieren, zeigte sich hier, daß Frauen im Vergleich zu Männern eine differenzierter strukturierte $\mathrm{ZO}$ nicht nur im familialen, sondern auch im Berufsbereich hatten. Diese Befunde deuten auf neue Probleme bei der Änderung der Geschlechtsrollenorientierung hin: Frauen - insbesondere Berufsschülerinnen - hatten ausgeprägter strukturierte Befürchtungen als Männer, daß sich ihre beruflichen Wünsche nicht erfüllen würden; auBerdem trauten sie sich weniger als Männer zu, aus eigener Kraft îre Hoffnungen erfüllen zu können. - Wenn also, anders als in der Literatur üblich, ZO thematisch und kognitiv-affektiv differenziert gemessen wird, zeigt sich, daß die Struktur der Zukunftsorientierung von Jugendlichen geschlechts- und bildungsabhängig ist. Diese Ergebnisse lassen sich - teilweise post-hoc - durchaus im Sinne traditionellen Geschlechtsrollenlernens und darüberhinaus als Merkmal der Orientierung an sozialen und ökologischen Gegebenheiten deuten.

\section{Einleitung}

Macht sich in der Zukunftsvorstellung von Jugendlichen ein Wandel der Geschlechtsrollenorientierung bemerkbar oder folgen Jugendliche in ihren Hoffnungen und Befürchtungen, in ihren Zukunftserwartungen und der Art, wie sie ihre Zukunft bewerten, weiterhin - wie vielfach in der Literatur belegt - den traditionellen Geschlechtsrollenmustern? Dieser Frage soll hier nachgegangen werden, indem zum einen der Bildungsstand der Jugendlichen als mögliche Einflußvariable auf die Zukunftsorientierung ( $\mathrm{ZO}$ ) mit berücksichtigt wird, und zum anderen, indem nicht, wie bisher in den meisten Untersuchungen üblich, das Konzept der ZO global, sondern hinsichtlich seiner kognitiv-affektiven und thematisch differenzierten Ausprägung bei männlichen und weiblichen Jugendlichen gemessen wird.

\section{Zukunftsorientierung (ZO) wird gemäß der}

Theorie der Zeitperspektive von Lewin (1948) und unter Bezug auf die Erwartungs-Wert-Theorien (z.B. Atkinson/Raynor 1974) als Antizipation und Bewertung zukünftiger Ereignisse verstanden (vgl. Trommsdorff et al. 1978). Damit sind verschiedene Aspekte der Zukunftsorientierung zu operationalisieren: die Differenziertheit der Antizipation zukünftiger Ereignisse, die eher positive oder negative Bewertung der Zukunft und die zugeschriebene Verursachung (eher durch Umwelt oder eher durch die eigene Person) erwarteter zukünftiger Entwicklungen.

Die Untersuchung von individuellen Differenzen in der Zukunftsorientierung kann zur Klärung der Bedeutung individueller, ,naiver“ Theorien für soziales Handeln beitragen: Die Art und Ausprägung der Zukunftsorientierung beschreibt, wie die Person ihre Zukunft antizipiert und bewertet und eigenes soziales Handeln in bezug auf die gegenwärtige und zukünftige Umwelt deutet.

Unterschiede in Art und Ausprägung der Zukunftsorientierung bei Frauen und Männern können - je nach theoretischer Präferenz - eher aufgrund von biologischen, sozialen und/oder kognitiv-motivationalen Merkmalen vorhergesagt und interpretiert werden (vgl. Maccoby/Jacklin 1974; Hetherington 1976; Sullerot 1979). In der vorliegenden Arbeit sollen soziale sowie kognitivmotivationale Faktoren für einen Erklärungsan-

1 Diese Arbeit ist im Sonderforschungsbereich 24, Sozial- und Wirtschaftspsychologische Entscheidungsforschung der Universität Mannheim, unter Verwendung der von der Deutschen Forschungsgemeinschaft und des Landes Baden-Württemberg zur Verfügung gestellten Mittel entstanden. Wir danken Wolfgang Woerner für seine Hilfe bei der Korrektur des Manuskriptes. 
satz geschlechtsspezifischer Differenzen in der Zukunftsorientierung herangezogen werden.

Hypothesen über geschlechtsspezifische Unterschiede in der Zukunftsorientierung lassen sich ableiten, wenn man von der Annahme ausgeht, daß Zukunftsorientierung - gerade auch unter Berücksichtigung ihrer Handlungswirksamkeit immer erfahrungsabhängig ist, also von dem Sozialisationsgeschehen, den bisher ausgeübten sozialen Rollen, der kognitiven und der motivationalen Entwicklung der Person abhängt. Dies ist empirisch in einigen Untersuchungen nachgewiesen worden (vgl. Klineberg 1968; Nuttin/Grommen 1975; Trommsdorff et al. 1979).

So ist also zu erwarten, daß die Geschlechtsrolle, die in jeder Kultur mit besonderen Erfahrungen verknüpft ist, die Zukunftsorientierung von Individuen entsprechend beeinflußt. Diese geschlechtsspezifischen Erfahrungen sind nicht unabhängig von sozialen Gegebenheiten, die den individuellen Handlungsspielraum, z.B. auf der Grundlage von ökologischen Faktoren, sozialem Status, Ausbildungsniveau und den damit verbundenen Berufsmöglichkeiten, mitbestimmen.

Wir wollen uns hier nur auf die Fragen beschränken, (a) wie die Zukunftsorientierung von jungen Frauen und Männern verschiedenen Bildungsstandes ausgeprägt ist und (b), ob unabhängig vom Bildungsstand geschlechtsspezifische Unterschiede in der Zukunftsorientierung gegeben sind bzw. in welcher Weise sie mit dem jeweiligen Bildungsstand zusammenhängen.

Zukunftsorientierung verstehen wir hier als thematisch differenzierte Antizipation und Bewertung der Zukunft. Zukunftsorientierung ist also keine globale Variable, sondern umfaßt themenspezifische kognitive und motivationale Aspekte (vgl. Trommsdorff/Lamm 1975). Wir untersuchen hier die Aspekte (a) Strukturierung (Differenziertheit von antizipierten Voraussetzungen für zukünftige Ereignisse), (b) Kausalattribuierung (Zuschreibung von Ursachen auf eher internale bzw. externale und stabile bzw. variable Faktoren), (c) Optimismus/Pessimismus (erwartete Wahrscheinlichkeit erhoffter bzw. be-

füchteter Ereignisse), (d) Wichtigkeit und (e) Erwünschtheit zukünftiger Ereignisse.
Geschlechtsspezifische Unterschiede. Gemäß traditionellen Geschlechtsmustern ist zu erwarten, daß Mädchen ihre Zukunft weniger als Jungen auf den Berufsbereich, aber dafür stärker auf den Bereich der Familie ausrichten. Der Mann hat in seiner Rolle als Haupternährer der Familie beruflich erfolgreich zu sein, während die Frau sich auf ihre zukünftige Rolle als Hausfrau und Mutter einstellt und davon ausgeht, einen Beruf nur vorübergehend auszuüben.

Gegen diese Vermutung spricht allerdings, daß die Emanzipationsbewegung der Frau und die Forderung nach gleicher Ausbildung und gleichen Berufschancen für Frauen und Männer sich auf die Zukunftsorientierung von Jugendlichen in der Weise auswirken müßten, daß heranwachsende Frauen und Männer hinsichtlich ihrer familialen und beruflichen Zukunft inzwischen ähnliche Deutungsmuster und Antizipationen entwickelt haben.

Solche Änderungen in den traditionellen Geschlechtsrolleneinstellungen sind jedoch vermutlich nicht unabhängig von dem Ausbildungsstand und den damit verbundenen Berufschancen des Jugendlichen. So wäre zu erwarten, daß sich Jugendliche aufgrund ihrer jeweils erfahrenen Schulbildung hinsichtlich ihrer Antizipation und Bewertung der Zukunft unterscheiden: Insbesondere solche Jugendliche, deren Berufschancen aufgrund einer qualifizierten Ausbildung besser sind, dürften keine oder bedeutend geringere geschlechtsspezifische Differenzen in der Zukunftsorientierung aufweisen als weniger qualifizierte Jugendliche.

Durch eine Reihe neuerer empirischer Befunde ist nachgewiesen worden, daß geschlechtsspezifische Differenzen hinsichtlich allgemeiner $\mathrm{Zu}$ kunftserwartungen und der Planung für Beruf und Familie im Sinne traditioneller Rollenvorstellungen noch bestehen (vgl. Lehr 1972; Rosenmayr/Kreutz 1973; Reitz 1974; Kreutz 1975); solche geschlechtsspezifischen Differenzen konnten wir auch in eigenen Untersuchungen iber verschiedene Bildungsgruppen Anfang der 70er Jahre bei Gymnasiasten und Hauptschülern nachweisen (vgl. Lamm et al. 1976).

Allerdings sprechen einige unserer Ergebnisse dafür, daß globale Hypothesen zur geschlechtsspe- 
zifischen Ausprägung (auch von thematisch differenzierten Komponenten) der Zukunftsorientierung zu differenzieren sind. In der zuletzt genannten Untersuchung von Lamm et al. (1976) zeigte sich nämlich, daß Mädchen hinsichtlich ihrer beruflichen Zukunft eher pessimistisch waren, Hauptschuler aber - wohl weil sie ihre Ziele niedriger als Gymnasiasten steckten - eher optimistisch in die Zukunft blickten. Auch waren Mädchen stärker als Jungen davon überzeugt, daß zukünftige Entwicklungen eher von externen Bedingungen abhängen als von ihnen selbst. Dagegen konnten wir nachweisen, daß Mädchen im Vergleich zu Jungen befürchtete berufliche Entwicklungen, also berufliches Versagen, eher sich selbst zuschreiben (vgl. Trommsdorff et al. 1979).

Diese Ergebnisse sprechen für eine größere $\mathrm{Miß}$ erfolgsängstlichkeit und mangelnde Zuversicht hinsichtlich der beruflichen Zukunft bei Mädchen im Vergleich zu Jungen und für ein - bei Mädchen - geringer ausgeprägtes Zutrauen in eigene Einflußmöglichkeiten. Aus Untersuchungen zur Leistungsmotivation läßt sich diese Deutung der Ergebnisse stützen; danach sind Mädchen oft unsicherer hinsichtlich ihrer Leistungserfolge im akademischen Bereich und vertrauen ihren Fähigkeiten weniger als Jungen (vgl. Horner 1972; Tresemer 1977).

In bezug auf konkrete Handlungskonsequenzen glauben Frauen eher an Glück und Zufall (für die Erklärung von Erfolg und Mißerfolg) als Männer (vgl. Feather 1969). Ähnlich konnten Deaux et al. (1975) nachweisen, daß Jungen mehr als Mädchen Geschicklichkeitsaufgaben bevorzugen, Mädchen dagegen eher Aufgaben bevorzugen, deren Erfolg vom Glück abhängt. Solche Präferenzen lassen sich als Folge subjektiver Einschätzung von Erfolgsaussichten deuten. Es ist bekannt, daß Jungen im Vergleich zu Mädchen optimistischer sind hinsichtlich ihrer Erfolgsaussichten in Leistungs- und Geschicklichkeitsaufgaben (vgl. Crandall 1969; Feather 1969).

Daher ist also anzunehmen, daß die Zukunftsorientierung von Mädchen stärker external als die von Jungen ist, und daß sich Mädchen im Leistungsbereich weniger zutrauen bzw. pessimistischere Erfolgserwartungen als Jungen haben.
Für unsere eher explorative Untersuchung wurden daher die folgenden Hypothesen formuliert:

(I). Gemäß herkömmlichen Geschlechtsrollenmustern wäre zu erwarten, daß Mädchen im Vergleich zu Jungen (1) hinsichtlich ihres Berufsbereiches ihre Zukunft (a) weniger strukturieren, (b) eher external attribuieren, (c) in diesem Bereich weniger Erfolge erhoffen und (d) diesen Bereich als weniger wichtig erachten, dagegen aber (2) den privaten und familialen $\mathrm{Be}$ reich (a) stärker strukturieren, (b) dort erfolgszuversichtlicher sind, (c) diesem Bereich mehr Bedeutung (Wichtigkeit) zumessen und (d) eher internal attribuieren. (II.) Bei niedrigerem Bildungsniveau müßten diese Differenzen (in Richtung auf ein stärker traditionelles Geschlechtsrollenbild) deutlicher ausgeprägt sein als bei höherem Bildungsstatus.

\section{Methode}

Stichprobe. Es wurde hier nicht nur intendiert, eine Bestandsaufnahme der $\mathrm{ZO}$ von Jugendlichen vorzunehmen, sondern darüberhinaus sollte exploriert werden, ob und welche theoretisch anzunehmenden Zusammenhänge zwischen Geschlecht, Bildung und $\mathrm{ZO}$ bestehen. Dazu wurde hier keine Repräsentativbefragung vorgenommen, sondern nur eine relativ kleine Stichprobe befragt. Damit ist die Generalisierbarkeit der Ergebnisse von vornherein eingeschränkt. An der Untersuchung nahmen 23 männliche und 50 weibliche Berufsschïler, 41 männliche und 63 weibliche Gymnasiasten und 43 männliche und 36 weibliche Studenten gegen ein Honorar (DM 10,-) teil. Alle Probanden waren zwischen 18 und 25 Jahre alt.

Instrument. Zur Messung der Zukunftsorientierung wurde ein Fragebogeninventar verwendet, das gemäß unseres theoretischen Konzepts die folgenden Komponenten der Zukunftsorientierung in ihrer thematischen Ausprägung erfaßt: (a) Strukturiertheit, (b) Kausalattribuierung, (c) Optimismus/Pessimismus, (d) Wichtigkeit und (e) Erwünschtheit (vgl. Füchsle et al. 1980).

Diese Fragebögen bestanden aus einer Reihe von Aussagen über zukïnftige Ereignisse, die sich auf verschiedene Bereiche des privaten Lebens be- 
zogen. (Z.B. Item 10: ,Ich werde mein Ausbildungsziel nicht erreichen." Item 33: „Ich werde meinen Traumberuf ausüben." Item 77: „Ich werde seelisch gesund sein.") Die Inhaltsbereiche wurden faktorenanalytisch -- für jede Komponente der Zukunftsorientierung - generiert. Sie umfassen im wesentlichen den beruflichen, materiellen, Familien- und Persönlichkeitsbereich. Die teststatistischen Gütekriterien (Trennschärfe und interne Konsistenz) für die Faktoren sind zufriedenstellend (vgl. Füchsle et al. 1980). Die einzelnen Komponenten der $\mathrm{ZO}$ wurden folgendermaBen operationalisiert:

(a) Kognitive Strukturiertheit wurde operationalisiert als Anzahl von genannten Voraussetzungen für das Eintreten der vorgegebenen Hoffnungen und Befürchtungen (also der positiven und negativen Zukunftsmerkmale) (41 Items). (b) Kausalattribuierung wurde auf der Grundlage des Attribuierungsschemas von Weiner (1974) gemessen: Die Pbn sollten zu jedem Item angeben, ob sie die dazu antizipierten Ursachen dem Zufall (external-variabel), äußeren Umständen (external-stabil), eigenen Fähigkeiten (internalstabil) oder eigener Anstrengung (internal-variabel) zuschreiben. Daraus wurde je ein Index für ,Internalität/Externalität“ (34 Items) und ,Stabilität/Variabilität“ (33 Items) gebildet. (c) $O p$ timismus/Pessimismus wurde operationalisiert als hohe Eintrittswahrscheinlichkeit bei erhofften (positiven) und niedrige Eintrittswahrscheinlichkeit bei befürchteten (negativen) Ereignissen (Optimismus) bzw. niedrige Eintrittswahrscheinlichkeit bei erhofften und hohe Eintrittswahrscheinlichkeit bei befürchteten Ereignissen (Pessimismus) (7-Punkte-Skala für 69 Items). (d) Die Wichtigkeit zukünftiger Ereignisse (72 Items) sowie (e) die Erwünschtheit (69 Items) wurden jeweils auf 7-Punkte-Skalen gemessen. In die Auswertung gingen die Mittelwerte der Items jedes thematischen Bereichs der einzelnen Komponenten der $\mathrm{ZO}$ ein.

\section{Ablauf. Die Untersuchung wurde in Gruppen} durchgefuhrt. Versuchsleiter waren weibliche und männliche Studenten. Die Pbn machten zuerst Angaben über ihre Person (Alter, Geschlecht etc.) und bearbeiteten dann nacheinander die einzelnen Fragebögen zu den Komponenten der $\mathrm{Zu}$ kunftsorientierung.

\section{Ergebnisse}

Die Auswertung der Daten erfolgte durch 2x3-faktorielle Varianzanalysen (Faktor A: Geschiecht; Faktor B: Bildung - Berufsschüler, Gymnasiasten, Studenten) mit anschließenden post-hoc-Vergleichen (vgl. Hays 1969) und Berechnung der ,simple effects" (F $F_{S}$, vgl. Winer 1962).

\section{Geschlechtsspezifische Unterschiede}

Kognitive Strukturierung: Junge Frauen nannten im Vergleich zu Männern mehr Voraussetzungen für das Eintreten von Hoffnungen in dem Bereich ,Berufliche Sicherheit und berufliches Vorwärtskommen" ( $F=11.04, \mathrm{df}=1 / 244, \mathrm{p}<.01)$ (vgl. Tabelle 1). Sie nannten ebenfalls mehr Voraussetzungen als junge Männer in den Bereichen „Harmonisches Privatleben“" (Befürchtungen) $(F=20.63$ bzw. $F=11.39$, df jeweils $=1 / 244$, $\mathrm{p}<.01)($ vgl. Tabelle 1).

Kausalattribuierung. Junge Frauen erwarteten im Vergleich zu jungen Männern häufiger externale Ursachen für ein ,,ausgefülltes und gesichertes Privatleben" (Hoffnungen) und für ,,berufliches Vorwärtskommen" (Hoffnungen) ( $F=4.12$, $F=5.80$; jeweils $\mathrm{df}=1 / 244, \mathrm{p}<.05)(\mathrm{vgl}$. Tabelle 2).

Erwünschtheit. Für junge Frauen waren Hoffnungen in bezug auf ,,berufliches Vorwärtskommen“ erwünschter als für junge Männer $(F=5.86$, df = $1 / 247, p<.05)$. Hoffnungen im Bereich ,Materieller Wohlstand " waren für Frauen weniger erwünscht als für junge Männer $(F=5.30$, df $=$ $1 / 247, \mathrm{p}<.05)$ (vgl. Tabelle 4). Befürchtungen in bezug auf ,Krankheit und bedrohtes Privatleben" waren für Frauen ebenfalls unerwünschter als für Männer ( $F=4.49$, df $=1 / 247, \mathrm{p}<.01$ ) (vgl. Tabelle 4).

Wichtigkeit. Für Frauen waren Hoffnungen in bezug auf ,berufliches Vorwärtskommen“ wichtiger als für Männer $(\mathrm{F}=8.49, \mathrm{df}=1 / 247, \mathrm{p}<$ .01) (vgl. Tabelle 5).

Optimismus/Pessimismus. Frauen waren pessimistischer als Männer in bezug auf ein ,,ausgefuiltes und gesichertes Privatleben und berufliches Vorwärtskommen" (Hoffnungen) sowie 
TABELLE 1 Mittelwerte der kognitiven Strukturierung zukünftiger Ereignisse

\begin{tabular}{|c|c|c|c|c|c|c|c|c|}
\hline \multirow{2}{*}{$\begin{array}{l}\text { Bildung } \\
\text { Geschlecht }\end{array}$} & \multicolumn{2}{|c|}{ Berufsschüler } & \multicolumn{2}{|c|}{ Gymnasiasten } & \multicolumn{2}{|c|}{ Studenten } & \multirow[b]{2}{*}{ F } & \multirow[b]{2}{*}{$F G$} \\
\hline & $\underset{\bar{x}}{\text { Männer }}$ & $\underset{\bar{x}}{\text { Frauen }}$ & $\underset{\bar{x}}{\text { Männer }}$ & $\underset{\bar{x}}{\text { Frauen }}$ & $\underset{\bar{x}}{\text { Männer }}$ & Frauen & & \\
\hline $\begin{array}{l}\text { Kognitive Strukturierung }{ }^{1} \\
\text { Hoffnungen }\end{array}$ & & & & & & & & \\
\hline $\begin{array}{l}\text { Berufliche Sicherheit } \\
\text { und Vorwärtskommen }\end{array}$ & 1.89 & 2.05 & 2.27 & 2.79 & 2.40 & 2.71 & $\begin{array}{l}\mathrm{F}_{\mathrm{A}}=11.04^{*} \\
\mathrm{~F}_{\mathrm{B}}=12.68^{*}\end{array}$ & $\begin{array}{l}1 / 244 \\
2 / 244\end{array}$ \\
\hline $\begin{array}{l}\text { Harmonisches } \\
\text { Privatleben }\end{array}$ & 2.27 & 2.65 & 2.71 & 3.32 & 2.77 & 3.20 & $\begin{array}{l}F_{A}=20.63^{*} \\
F_{B}=10.30^{*}\end{array}$ & $\begin{array}{l}1 / 244 \\
2 / 244\end{array}$ \\
\hline $\begin{array}{c}\text { Befürchtungen } \\
\text { Bedrohtes } \\
\text { Privatleben }\end{array}$ & 2.21 & 2.35 & 2.56 & 3.17 & 2.69 & 2.98 & $\begin{array}{l}\mathrm{F}_{\mathrm{A}}=11.39^{*} \\
\mathrm{~F}_{\mathrm{B}}=12.07^{*}\end{array}$ & $\begin{array}{l}1 / 244 \\
2 / 244\end{array}$ \\
\hline
\end{tabular}

$* p \leqslant .01$.

$F_{A}=$ Geschlecht $; F_{B}=$ Bildung $; F G=$ Freiheitsgrade

1 Anzahl genannter Voraussetzungen je Bereich

TABELLE 2 Kausalattribuierung (external/internal) zukünftiger Ereignisse

\begin{tabular}{|c|c|c|c|c|c|c|c|c|}
\hline \multirow{2}{*}{$\begin{array}{l}\text { Bildung } \\
\text { Geschlecht }\end{array}$} & \multicolumn{2}{|c|}{ Berufsschüler } & \multicolumn{2}{|c|}{ Gymnasiasten } & \multicolumn{2}{|c|}{ Studenten } & \multirow[b]{2}{*}{ F } & \multirow[b]{2}{*}{ FG } \\
\hline & $\underset{\bar{x}}{\text { Männer }}$ & $\underset{\bar{x}}{\text { Frauen }}$ & $\underset{\bar{x}}{\text { Männer }}$ & $\underset{\bar{x}}{\text { Frauen }}$ & $\underset{\bar{x}}{\text { Männer }}$ & $\underset{\vec{x}}{\text { Frauen }}$ & & \\
\hline \multicolumn{9}{|l|}{$\begin{array}{l}\text { Kausalattribuierung } \\
\text { (external/internal) }\end{array}$} \\
\hline \multicolumn{9}{|l|}{ Hoffnungen } \\
\hline \multicolumn{9}{|l|}{$\begin{array}{l}\text { Ausgefülltes und } \\
\text { gesichertes Privat- }\end{array}$} \\
\hline $\begin{array}{l}\text { leben } \\
\text { Berufliches }\end{array}$ & 1.61 & 1.47 & 1.45 & 1.44 & 1.39 & 1.34 & $\begin{array}{l}\mathrm{F}_{\mathrm{A}}=4.12 * \\
\mathrm{~F}_{\mathrm{B}}=7.02 * *\end{array}$ & $\begin{array}{l}1 / 244 \\
2 / 244\end{array}$ \\
\hline Vorwärtskommen & 1.81 & 1.68 & 1.66 & 1.65 & 1.64 & 1.56 & $\begin{array}{l}\mathrm{F}_{\mathrm{A}}=5.80^{*} \\
\mathrm{~F}_{\mathrm{B}}=6.72^{* *}\end{array}$ & $\begin{array}{l}1 / 244 \\
2 / 244\end{array}$ \\
\hline \multicolumn{9}{|l|}{$\begin{array}{l}\text { Harmonische } \\
\text { familiäre }\end{array}$} \\
\hline Beziehungen & 1.91 & 1.92 & 1.84 & 1.83 & 1.82 & 1.78 & $\begin{array}{l}\mathrm{F}_{\mathrm{A}}=0.17 \\
\mathrm{~F}_{\mathrm{B}}=4.71 * *\end{array}$ & $\begin{array}{l}1 / 244 \\
2 / 244\end{array}$ \\
\hline \multicolumn{9}{|l|}{ Befürchtungen } \\
\hline Berufliches Versagen & 1.72 & 1.68 & 1.54 & 1.69 & 1.56 & 1.56 & $\begin{array}{l}F_{A}=0.93 \\
F_{B}=3.95^{*}\end{array}$ & $\begin{array}{l}1 / 244 \\
2 / 244\end{array}$ \\
\hline Bedrohtes Privatleben & 1.59 & 1.34 & 1.30 & 1.32 & 1.24 & 1.26 & $\begin{array}{l}\mathrm{F}_{\mathrm{A}}=3.40 \\
\mathrm{~F}_{\mathrm{B}}=10.78^{* *} \\
\mathrm{~F}_{\mathrm{AB}}=5.39^{* *}\end{array}$ & $\begin{array}{l}1 / 244 \\
2 / 244 \\
2 / 244\end{array}$ \\
\hline
\end{tabular}

${ }^{*} \mathrm{p} \leqslant .05 ; * * \mathrm{p} \leqslant .01$.

$F_{A}=$ Geschlecht $; F_{B}=$ Bildung; $F_{A B}=$ Geschlecht $x$ Bildung; $F G=$ Freiheit sgrade

${ }_{1}=$ external; 2 = internal 
TABELLE 3 Kausalattribuierung (variabel/stabil) zukünftiger Ereignisse

\begin{tabular}{|c|c|c|c|c|c|c|c|c|}
\hline \multirow{2}{*}{$\begin{array}{l}\text { Bildung } \\
\text { Geschlecht }\end{array}$} & \multicolumn{2}{|c|}{ Berufsschüler } & \multicolumn{2}{|c|}{ Gymnasiasten } & \multicolumn{2}{|c|}{ Studenten } & \multirow[b]{2}{*}{$\mathrm{F}$} & \multirow[b]{2}{*}{ FG } \\
\hline & $\underset{\bar{x}}{\operatorname{Männer~}}$ & $\underset{\bar{x}}{\text { Frauen }}$ & $\underset{\bar{x}}{\operatorname{Männer~}}$ & Frauen & $\underset{\bar{x}}{\operatorname{Männer~}}$ & Frauen & & \\
\hline \multicolumn{9}{|l|}{$\begin{array}{l}\text { Kausalattribuierung } \\
\text { (variabel/stabil) }^{1}\end{array}$} \\
\hline \multicolumn{9}{|l|}{$\begin{array}{l}\text { Ausgefülltes, gesichertes } \\
\text { und harmonisches }\end{array}$} \\
\hline Brivatleben & 1.51 & 1.50 & 1.56 & 1.54 & 1.56 & 1.60 & $\begin{array}{l}F_{A}=0.00 \\
F_{B}=1.66\end{array}$ & $\begin{array}{l}1 / 247 \\
2 / 247\end{array}$ \\
\hline $\begin{array}{l}\text { Berufliches } \\
\text { Vorwärtskommen }\end{array}$ & 1.51 & 1.62 & 1.57 & 1.54 & 1.58 & 1.63 & $\begin{array}{l}\mathrm{F}_{\mathrm{A}}=1.94 \\
\mathrm{~F}_{\mathrm{B}}=1.03\end{array}$ & $\begin{array}{l}1 / 247 \\
2 / 247\end{array}$ \\
\hline $\begin{array}{l}\text { Befürchtungen } \\
\text { Beruftiches Versagen }\end{array}$ & 1.73 & 1.61 & 1.48 & 1.52 & 1.40 & 1.50 & $\begin{array}{l}\mathrm{F}_{\mathrm{A}}=0.06 \\
\mathrm{~F}_{\mathrm{B}}=13.10^{* *} \\
\mathrm{~F}_{\mathrm{AB}}=3.23^{*}\end{array}$ & $\begin{array}{l}1 / 247 \\
2 / 247 \\
2 / 247\end{array}$ \\
\hline Bedrohtes Privatleben & 1.34 & 1.38 & 1.37 & 1.33 & 1.34 & 1.39 & $\begin{array}{l}\mathrm{F}_{\mathrm{A}}=0.35 \\
\mathrm{~F}_{\mathrm{B}}=0.10\end{array}$ & $\begin{array}{l}1 / 247 \\
2 / 247\end{array}$ \\
\hline
\end{tabular}

${ }^{*} \mathrm{p} \leqslant .05 ;{ }^{* *} \mathrm{p} \leqslant .01$.

$F_{A}=$ Geschlecht $; F_{B}=$ Bildung; $F_{A B}=$ Geschlecht $\times$ Bildung; $F G=$ Freiheitsgrade

$1_{1}=$ variabel: 2 = stabil

TABELLE 4 Erwünschtheit zukünftiger Ereignisse

\begin{tabular}{|c|c|c|c|c|c|c|c|c|}
\hline \multirow{2}{*}{$\begin{array}{l}\text { Bildung } \\
\text { Geschlecht }\end{array}$} & \multicolumn{2}{|c|}{ Berufsschüler } & \multicolumn{2}{|c|}{ Gymnasiasten } & \multicolumn{2}{|c|}{ Studenten } & \multirow[b]{2}{*}{$\mathrm{F}$} & \multirow[b]{2}{*}{ FG } \\
\hline & $\underset{\bar{x}}{\operatorname{Männer}}$ & $\underset{\bar{x}}{\text { Frauen }}$ & $\underset{\bar{x}}{\operatorname{Männer~}}$ & $\underset{\bar{x}}{\text { Frauen }}$ & $\underset{\bar{x}}{\operatorname{Männer}}$ & $\underset{\bar{x}}{\text { Frauen }}$ & & \\
\hline \multicolumn{9}{|l|}{ Erwünschtheit ${ }^{1}$} \\
\hline \multirow{2}{*}{\multicolumn{9}{|c|}{$\begin{array}{l}\text { Hoffnungen } \\
\text { Berufliches }\end{array}$}} \\
\hline & & & & & & & & \\
\hline & 0.30 & 0.30 & 0.29 & 6.43 & 6.20 & 6.48 & $\begin{array}{l}\mathrm{F}_{\mathrm{A}}=5.86^{*} \\
\mathrm{~F}_{\mathrm{B}}=2.10\end{array}$ & $\begin{array}{l}1 / 247 \\
2 / 247\end{array}$ \\
\hline Materieller Wohlstand & 6.22 & 6.20 & 6.00 & 5.52 & 5.47 & 5.32 & $\begin{array}{l}\mathrm{F}_{\mathrm{A}}=5.30^{*} \\
\mathrm{~F}_{\mathrm{B}}=22.52 * *\end{array}$ & $\begin{array}{l}1 / 247 \\
2 / 247\end{array}$ \\
\hline \multicolumn{9}{|l|}{ Harmonische familiäre } \\
\hline Beziehungen & 6.39 & 6.44 & 5.93 & 6.02 & 5.78 & 5.74 & $\begin{array}{l}\mathrm{F}_{\mathrm{A}}=0.13 \\
\mathrm{~F}_{\mathrm{B}}=18.49 * *\end{array}$ & $\begin{array}{l}1 / 247 \\
2 / 247\end{array}$ \\
\hline \multicolumn{9}{|l|}{ Befürchtungen } \\
\hline $\begin{array}{l}\text { Krankheit und } \\
\text { bedrohtes Privatleben }\end{array}$ & 1.50 & 1.41 & 1.77 & 1.55 & 1.81 & 1.61 & $\begin{array}{l}\mathrm{F}_{\mathrm{A}}=4.49 * \\
\mathrm{~F}_{\mathrm{B}}=3.39^{*}\end{array}$ & $\begin{array}{l}1 / 247 \\
2 / 247\end{array}$ \\
\hline $\begin{array}{l}\text { Berufliches Versagen und } \\
\text { materielle Unsicherheit }\end{array}$ & 1.37 & 1.47 & 1.86 & 1.73 & 1.94 & 1.80 & $\begin{array}{l}\mathrm{F}_{\mathrm{A}}=0.48 \\
\mathrm{~F}_{\mathrm{B}}=10.77 * *\end{array}$ & $\begin{array}{l}1 / 247 \\
2 / 247\end{array}$ \\
\hline
\end{tabular}

${ }^{*} \mathrm{p} \leqslant .05 ;{ }^{* *} \mathrm{p} \leqslant .01$.

$\mathrm{F}_{\mathrm{A}}=$ Geschlecht $\mathrm{F}_{\mathrm{B}}=$ Bildung; $\mathrm{FG}=$ Freiheitsgrade

1 Skala von $1=$ sehr unerwünscht bis $7=$ sehr erwünscht 
TABELLE 5 Wichtigkeit zukünftiger Ereignisse

\begin{tabular}{|c|c|c|c|c|c|c|c|c|}
\hline \multirow{2}{*}{$\begin{array}{l}\text { Bildung } \\
\text { Geschlecht }\end{array}$} & \multicolumn{2}{|c|}{ Berufsschüler } & \multicolumn{2}{|c|}{ Gymnasiasten } & \multicolumn{2}{|c|}{ Studenten } & \multirow[b]{2}{*}{$\mathrm{F}$} & \multirow[b]{2}{*}{ FG } \\
\hline & $\underset{\bar{x}}{\text { Männer }}$ & Frauen & $\underset{\bar{x}}{\operatorname{Männer}}$ & $\underset{\bar{x}}{\text { Frauen }}$ & $\underset{\bar{x}}{\text { Männer }}$ & $\underset{\bar{x}}{\text { Frauen }}$ & & \\
\hline \multicolumn{9}{|l|}{ Wichtigkeit $^{1}$} \\
\hline \multicolumn{9}{|l|}{$\begin{array}{l}\text { Hoffrungen } \\
\text { Berufliches }\end{array}$} \\
\hline Vorwärtskommen & 6.05 & 6.29 & 5.98 & 6.22 & 5.85 & 6.26 & $\begin{array}{l}F_{A}=8.49 * \\
F_{B}=0.37\end{array}$ & $\begin{array}{l}1 / 247 \\
2 / 247\end{array}$ \\
\hline Materieller Wohlstand & 5.68 & 5.70 & 5.54 & 5.00 & 4.81 & 4.82 & $\begin{array}{l}\mathrm{F}_{\mathrm{A}}=1.76 \\
\mathrm{~F}_{\mathrm{B}}=14.14^{*}\end{array}$ & $\begin{array}{l}1 / 250 \\
2 / 250\end{array}$ \\
\hline $\begin{array}{l}\text { Harmonische familiäre } \\
\text { Beziehungen }\end{array}$ & 6.19 & 6.47 & 5.97 & 5.97 & 5.61 & 5.74 & $\begin{array}{l}\mathrm{F}_{\mathrm{A}}=1.46 \\
\mathrm{~F}_{\mathrm{B}}=9.64^{*}\end{array}$ & $\begin{array}{l}1 / 250 \\
2 / 250\end{array}$ \\
\hline $\begin{array}{l}\text { Befürchtungen } \\
\text { Krankheit und } \\
\text { bedrohtes Privatleben }\end{array}$ & 4.00 & 4.83 & 4.27 & 4.90 & 4.36 & 4.34 & $\begin{array}{l}\mathrm{F}_{\mathrm{A}}=3.20 \\
\mathrm{~F}_{\mathrm{B}}=0.32\end{array}$ & $\begin{array}{l}1 / 250 \\
2 / 250\end{array}$ \\
\hline Materielle Unsicherheit & 4.00 & 4.56 & 4.29 & 4.44 & 4.26 & 4.02 & $\begin{array}{l}\mathrm{F}_{\mathrm{A}}=0.51 \\
\mathrm{~F}_{\mathrm{B}}=0.40\end{array}$ & $\begin{array}{l}1 / 250 \\
2 / 250\end{array}$ \\
\hline
\end{tabular}

$* p \leqslant .01$.

$F_{A}=$ Geschlecht $; F_{B}=$ Bildung; $F G=$ Freiheitsgrade

${ }^{1}$ Skala von $1=$ äußerst unwichtig bis $7=$ äußerst wichtig

TABELLE 6 Optimismus/Pessimismus hinsichtlich zukünftiger Ereignisse

\begin{tabular}{|c|c|c|c|c|c|c|c|c|}
\hline \multirow{2}{*}{$\begin{array}{l}\text { Bildung } \\
\text { Geschlecht }\end{array}$} & \multicolumn{2}{|c|}{ Berufsschüler } & \multicolumn{2}{|c|}{ Gymnasiasten } & \multicolumn{2}{|c|}{ Studenten } & \multirow[b]{2}{*}{$\mathbf{F}$} & \multirow[b]{2}{*}{ FG } \\
\hline & Männer & $\underset{\bar{x}}{\text { Frauen }}$ & $\underset{\bar{x}}{\text { Männer }}$ & $\underset{\overrightarrow{\mathrm{x}}}{\text { Frauen }}$ & $\underset{\bar{x}}{\text { Männer }}$ & $\underset{\bar{x}}{\text { Frauen }}$ & & \\
\hline \\
\hline \multicolumn{9}{|l|}{$\begin{array}{l}\text { Hoffnungen } \\
\text { Ausgefülltes und } \\
\text { gesichertes Privatleben; } \\
\text { berufliches Vorwärts- }\end{array}$} \\
\hline kommen & 5.33 & 4.74 & 4.80 & 4.86 & 4.76 & 4.63 & $\begin{array}{l}F_{A}=6.60^{*} \\
F_{B}=4.95^{* *} \\
F_{A B}=5.01^{* *}\end{array}$ & $\begin{array}{l}1 / 250 \\
2 / 250 \\
2 / 250\end{array}$ \\
\hline \multicolumn{9}{|l|}{ Harmonische familiäre } \\
\hline Beziehungen & 5.57 & 5.13 & 4.94 & 5.00 & 4.73 & 4.61 & $\begin{array}{l}\mathrm{F}_{\mathrm{A}}=3.10 \\
\mathrm{~F}_{\mathrm{B}}=15.60^{* *}\end{array}$ & $\begin{array}{l}1 / 250 \\
2 / 250\end{array}$ \\
\hline Materieller Wohlstand & 5.52 & 4.96 & 5.15 & 5.00 & 5.11 & 4.73 & $\begin{array}{l}\mathrm{F}_{\mathrm{A}}=12.55^{* *} \\
\mathrm{~F}_{\mathrm{B}}=3.03^{*}\end{array}$ & $\begin{array}{l}1 / 250 \\
2 / 250\end{array}$ \\
\hline \multicolumn{9}{|l|}{ Befürchtungen } \\
\hline $\begin{array}{l}\text { Berufliches Versagen und } \\
\text { materielle Unsicherheit }\end{array}$ & 2.70 & 3.12 & 3.30 & 3.32 & 3.13 & 3.49 & $\begin{array}{l}\mathrm{F}_{\mathrm{A}}=8.26^{* *} \\
\mathrm{~F}_{\mathrm{B}}=7.28^{* *}\end{array}$ & $\begin{array}{l}1 / 250 \\
2 / 250\end{array}$ \\
\hline Krankheit und bedrohtes & & & & & & & & \\
\hline Privatleben & 3.26 & 3.42 & 3.37 & 3.60 & 3.51 & 3.48 & $\begin{array}{l}\mathrm{F}_{\mathrm{A}}=2.01 \\
\mathrm{~F}_{\mathrm{B}}=1.20\end{array}$ & $\begin{array}{l}1 / 250 \\
2 / 250\end{array}$ \\
\hline
\end{tabular}

${ }^{*} \mathrm{p} \leqslant .05 ;{ }^{* *} \mathrm{p} \leqslant .01$.

$F_{A}=$ Geschlecht $; F_{B}=$ Bildung $; F_{A B}=$ Geschlecht $x$ Bildung; $F G=$ Freiheitsgrade

1 Skalen jeweils von $1=$ ausgeschlossen bis $7=$ sehr sicher 
in bezug auf ,materiellen Wohlstand" (Hoffnungen $)(F=6.60, F=12.55$; df jeweils $1 / 250$, $\mathrm{p}<.05)(\mathrm{vgl}$. Tabelle 6). Frauen waren pessimistischer als Männer in bezug auf ,berufliches Versagen und materielle Unsicherheit" (Befürchtungen $)(F=8.26, d f=1 / 250, p<.01)(v g l$. Tabelle 6).

\section{Bildungseffekte}

Kognitive Strukturierung. Berufsschüler nannten weniger Voraussetzungen für Hoffnungen für ein ,harmonisches Privatleben“, für „,berufliche Sicherheit, berufliches Vorwärtskommen" sowie in bezug auf ein „bedrohtes Privatleben“ als Gymnasiasten und Studenten $(\mathrm{F}=10.30, \mathrm{~F}=$ $12.68, F=12.07$; jeweils $\mathrm{df}=2 / 244, \mathrm{p}<.01)$ (vgl. Tabelle 1).

Kausalattribuierung. Berufsschüler erwarteten häufiger als Studenten, daß das Eintreten ihrer Hoffnungen in bezug auf ihr ,, berufliches Vorwärtskommen" sowie ,,ausgefülltes und gesichertes Privatleben" und ,harmonische familiäre Beziehungen" durch eigene Einflüsse realisiert werden würde $(F=6.72, F=7.02, F=4.71$; jeweils $\mathrm{df}=2 / 244, \mathrm{p}<.01)(\mathrm{vgl}$. Tabelle 2$)$.

Hinsichtlich des Eintretens ihrer Befürchtungen für ein ,bedrohtes Privatleben“ und ,berufliches Versagen" fühlten sich Berufsschüler häufiger selbst verantwortlich als Studenten (jeweils $\mathrm{p}<$ $.05)$.

Berufsschüler glaubten in bezug auf das Eintreten ,beruflichen Versagens" mehr an stabile Ursachen als Gymnasiasten und Studenten $(\mathrm{F}=$ $13.10, \mathrm{df}=2 / 244, \mathrm{p}<.01)($ vgl. Tabelle 2$)$.

Erwünschtheit. Erhoffter ,,materieller Wohlstand“ und ,harmonisches Familienleben" waren für Berufsschüler erwünschter als für Gymnasiasten und Studenten $(F=22.52, F=18.49 ; \mathrm{df}=$ $2 / 247, \mathrm{p}<.01$ ). Befürchtete Entwicklungen hinsichtlich ,,beruflichem Versagen und materieller Unsicherheit" waren für Berufsschüler unerwünschter als für Gymnasiasten und Studenten $(\mathrm{F}=10.77 ; \mathrm{df}=2 / 247, \mathrm{p}<.01)($ vgl. Tabelle 4$)$.

Wichtigkeit. Für Berufsschüler war das Eintreten von Hoffnungen in bezug auf ,,materiellen Wohlstand" und in bezug auf ,harmonische familiäre
Beziehungen" wichtiger als für Gymnasiasten und Studenten $(F=14.14, F=9.64 ; \mathrm{df}=2 / 250$ $\mathrm{p}<.01)$.

Optimismus/Pessimismus. Berufsschüler waren in bezug auf erhoffte ,harmonische familiäre Beziehungen "optimistischer als Studenten $(\mathrm{F}=$ 15.60 , df $=2 / 250, p<.01$ ).

\section{Wechselwirkungen zwischen Geschlecht und Bildung}

Die Überprïfung der Einfacheffekte zeigte, daß der Haupteffekt des Bildungsfaktors bei der $A t$ tribuienung des „bedrohten Privatlebens" auf eine stärker internale Attribuierung der männlichen Berufsschüler zurückzuführen war $\left(\mathrm{F}_{\mathrm{S}}=\right.$ 11.81 , df $=2 / 244, \mathrm{p}<.01)$. Der Geschlechtseffekt war darauf zurückzuführen, daß vor allem die männlichen Berufsschüler internaler attribuierten als die weiblichen Berufsschüler $\left(\mathrm{F}_{\mathrm{S}}=\right.$ 12.23, $\mathrm{df}=1 / 244, \mathrm{p}<.01)($ vgl. Tabelle 2$)$.

Der Bildungseffekt bei der Stabilitäts-/Variabilitätsattribuierung von befürchtetem ,,beruflichen Versagen “ beruhte darauf, daß männliche Berufsschüler häufiger stabile Ursachen antizipierten als die übrigen Gruppen $\left(F_{S}=11.41\right.$, $d f=2 / 247$, $\mathrm{p}<.01)$ (vgl. Tabelle 3).

Der Geschlechtseffekt in bezug auf Optimismus im Bereich ,Ausgefülltes und gesichertes Privatleben und berufliches Vorwärtskommen" beruhte auf Unterschieden zwischen den Berufsschülern: Die männlichen Berufsschüler waren optimistischer als die weiblichen $\left(F_{S}=13.00, \mathrm{df}=\right.$ $1 / 250, \mathrm{p}<.01)(\mathrm{vgl}$. Tabelle 6).

\section{Diskussion}

Die angenommenen Unterschiede in der $\mathrm{Zu}$ kunftsorientierung von weiblichen und männlichen Jugendlichen verschiedenen Bildungsstandes wurden durch die Ergebnisse nur teilweise belegt. Darüber hinaus ergaben sich interessante Befunde, die nicht vorhergesagt worden waren, die aber beim Versuch ihrer post-hoc Erklärung eine Reihe interessanter Fragen aufwarfen, denen in weiteren Untersuchungen nachgegangen werden müßte. 
Insgesamt zeigen die Resultate, daß Mädchen -. wie vorhergesagt - ihre private, aber entgegen unserer Hypothese auch ihre berufliche $\mathrm{Zu}$ kunft differenzierter als Jungen strukturieren und nicht nur wie erwartet im beruflichen, sondern auch im privaten Bereich weniger an eigene Einflußmöglichkeiten glauben, weniger optimistisch hinsichtlich des Eintretens erhoffter bzw. befürchteter Entwicklungen sind und nicht dem familialen, sondern dem beruflichen Bereich ein nicht vorhergesagtes größeres Gewicht (Erwünschtheit und Wichtigkeit) geben als Jungen. Einige dieser Ergebnisse (zur Attribuierung und zu Optimismus/Pessimismus) beruhen z.T. auf Interaktionseffekten mit dem Bildungsstand. Nicht vorhergesagte Bildungs-Haupteffekte zeigen, daß Berufsschüler ihre Zukunft weniger strukturieren, häufiger an stabilen und eigenen Einfluß glauben, optimistischer sind und dem Bereich materiellen Wohlstands mehr Gewicht als Gymnasiasten und Studenten geben.

Zunächst einmal ist festzuhalten, daß die $\mathrm{Zu}$ kunftsorientierung der hier befragten Frauen keineswegs so einfach in der Weise durch traditionelle Geschlechtsrollenvorstellungen geprägt ist, daß ihnen etwa ihre berufliche Zukunft weniger bedeutet als den Männern. Ganz im Gegenteil deuten unsere Ergebnisse darauf hin, daß Frauen ihrer beruflichen Zukunft weitaus mehr Bedeutung zumessen als Männer. Ihr erhofftes berufliches Vorwärtskommen ist ihnen offenbar zudem wesentlich erwünschter und wichtiger als den Männern.

Beruflicher Erfolg bedeutet für Frauen jedoch nicht unbedingt auch materieller Erfolg und höheres Einkommen. Materieller Erfolg ist für die Frauen weniger bedeutsam bzw. erwünscht als für Männer. Allerdings sind Frauen weniger zuversichtlich als Männer, daß sie beruflich erfolgreich sein können und materielle Sicherheit erlangen werden. Im Vergleich zu Männern trauen sie sich selbst weniger $\mathrm{zu}$, die Realisierung ihrer Hoffnungen bezüglich ihres beruflichen Erfolges selbst steuern zu können: Sie machen eher Zufall und Glück für mögliche Erfolge verantwortlich.

Unsere ursprüngliche Hypothesen müssen aufgrund der Ergebnisse also differenziert werden: Einerseits ist den Frauen mehr als den Männern ihr beruflicher Erfolg bedeutsam und erwünscht, andererseits sind sie eher pessimistisch und unsicher, daß sich ihre Hoffnungen überhaupt und durch ihr eigenes Zutun erfüllen können.

In dieses Bild lassen sich sehr gut die Ergebnisse zur Strukturierung der Zukunft einfügen: Frauen antizipieren mehr Voraussetzungen für ihre berufliche Sicherheit und Vorwärtskommen als Männer. Dieses Thema hat für sie, wie wir gesehen haben, auch eine größere Bedeutung. Gleichzeitig scheinen ihnen diese Zukunftsaspekte vergleichsweise mehr Unsicherheit zu vermitteln. Die von ihnen genannten Voraussetzungen für die Realisierung ihrer Hoffnungen im beruflichen Bereich sind vermutlich auch deshalb stärker differenziert als bei Männern, weil diese Voraussetzungen Schwierigkeiten darstellen können, die überwunden werden müßten, um den von ihnen gewünschten beruflichen Erfolg überhaupt erreichen zu können. Daraus läßt sich schließen, daß sie im Vergleich zu Männern eher Schwierigkeiten und Hindernisse auf dem Weg der beruflichen Karriere antizipieren.

Der berufliche Bereich macht jedoch nur einen thematischen Aspekt der Zukunftsorientierung aus. Frauen und Männer strukturieren ihre $\mathrm{Zu}$ kunft auch hinsichtlich des privaten Bereichs und familialer und persönlicher Hoffnungen und Befürchtungen unterschiedlich. Hier zeigt sich nun nicht etwa, daß Frauen mit ihren auf beruflichen Erfolg ausgerichteten Hoffnungen und $\mathrm{Be}$ fürchtungen den persönlichen und privaten $\mathrm{Be}$ reich vernachlässigen und/oder die Erfuillung persönlicher Hoffnungen für selbstverständlich halten. Im Gegenteil, sie sind auch hier weniger optimistisch als Männer, daß sich in diesem Bereich ihre Hoffnungen erfüllen werden. Befürchtete Entwicklungen im Privatbereich sind fur sie vermutlich bedrohlicher (unerwünschter) als für Männer, und sie trauen sich auch hier weniger eigene Einflußmöglichkeiten zu, ihre Hoffnungen in diesem Bereich zu realisieren. Mit dieser posthoc Interpretation stimmt auch überein, daß mehr Voraussetzungen bzw. Hindernisse für die Realisierung des von ihnen erhofften harmonischen Privatlebens antizipiert werden als von Männern.

Hier zeichnet sich ein Bild der Zukunftsorientierung von jungen Frauen und Männern ab, welches die Annahme zuläßt, daß Frauen in den zentralen Bereichen ihrer Lebenserfüllung weni- 
ger zuversichtlich als Männer in die Zukunft blicken. Vielmehr scheinen sie im Vergleich zu Männern zu fürchten, daß sich ihre Hoffnungen nicht erfullen und daß ihre Befürchtungen eintreten werden, ohne aus eigener Kraft viel gegen die zahlreichen Schwierigkeiten und Hindernisse ausrichten zu können. Diese pessimistische und external attribuierende Haltung nehmen Frauen sowohl hinsichtlich ihrer persönlich-privaten als auch hinsichtlich ihrer beruflichen Zukunft deutlich stärker ein als Männer. Damit deuten die Ergebnisse auf eine vergleichsweise größere Mißerfolgsängstlichkeit bzw. größeren Pessimismus bei Frauen hin und drücken ein bei ihnen geringeres Zutrauen in eigene Einflußmöglichkeiten hinsichtlich zukünftiger beruflicher Erfolge aus.

Tatsächlich ist aus der Literatur bekannt, daß solche Stereotype hinsichtlich der Attribuierungspräferenz von Jungen und Mädchen oder Frauen und Männern durchaus vertreten werden. Fragt man Studenten nach der idealen Frau bzw. dem idealen Mann, so schreiben sie Frauen eher eine externale und Männern eher eine internale Orientierung zu (vgl. Hochreich 1975). Wie be. reits oben berichtet, wurden solche Geschlechtsdifferenzen durch empirische Untersuchungen von tatsächlichen Erfolgsschätzungen (vgl. Crandall 1969; Feather 1969) und Handlungspräferenzen (vgl. Deaux et al. 1975) auch nachgewiesen.

Kann man also diese Ergebnisse einfach auf vorgegebene geschlechtsspezifische Persönlichkeitsmerkmale zurückführen und behaupten, daß der weniger ausgeprägte Optimismus der Frauen fur eine generelle geschlechtsspezifische größere Unsicherheit spricht, die ausgeprägtere externale Attribuierung für eine generelle Schicksalsgläubigkeit, die stärker strukturierten Antizipationen von Hindernissen für ängstliche Besorgnis, die größere Wichtigkeit und Erwünschtheit der familialen und beruflichen Zukunft für eine ausgeprägtere Motivation und größere emotionale Engagiertheit? Solche Interpretationen erscheinen doch zu einfach und - nach den Daten dieser Untersuchung - so undifferenziert empirisch nicht haltbar.

Dies wird u.a. dadurch deutlich, daß Geschlechtseffekte nicht unabhängig vom Bildungsstand der jungen Frauen und Männer sind: Der größere Optimismus hinsichtlich des beruflichen Vorwärtskommens und ausgefüllten Privatlebens von Männern im Vergleich zu Frauen zeigt sich nur bei der Gruppe der Berufsschüler, also nicht bei Gymnasiasten und Studenten. Ebenso ist die größere Überzeugung von externalen Einflüssen, die Frauen im Bereich befürchteter Entwicklungen im Privatleben zeigen, nur in der Gruppe der Berufsschüler festzustellen.

Geringerer Optimismus und stärker externale Attribuierung in einigen Bereichen unterscheiden also die bildungsschwächeren Frauen und Männer voneinander. Die wohl tatsächlich geringeren beruflichen Erfolgschancen werden also offenbar von den Berufsschülerinnen relativ realistisch eingeschätzt. Angesichts der Arbeitsmarktlage bewerten sie vermutlich ihr Geschlecht und ihre Qualifikation als Faktoren, die ungünstige Aussichten für berufliche Sicherheit und Karriere vermitteln. Zusätzlich läßt die antizipierte Inanspruchnahme durch die spätere Rollenübernahme der Hausfrau und Mutter Hoffnungen für ein berufliches Vorwärtskommen auf lange Sicht unrealistisch scheinen.

In dieser Hinsicht sind die hier befragten Berufsschülerinnen weitaus realistischer als die um 4-6 Jahre jüngeren Hauptschülerinnen der Untersuchung von Lamm et al. (1976). Die Hauptschülerinnen hatten vermutlich zu dem Zeit. punkt der Befragung noch so gut wie keine praktischen Kenntnisse und konkreten Informationen hinsichtlich ihrer tatsächlichen Weiterbildungs- und Berufsmöglichkeiten und damit verbundenen Erfolgschancen. Vielleicht war den damals von uns befragten Hauptschülerinnen ihre berufliche und private Zukunft so unklar (die Genauigkeit ihrer Zukunftsstrukturierung war ausgesprochen gering im Vergleich zu den anderen Gruppen), daß sie sich ohne realitätsbezogene Einschränkung dem Wunschdenken und einem überhöhten Optimismus überlassen konnten. Die Berufsschülerinnen der vorliegenden Befragung dagegen verfügen über einige Jahre mehr Lebens- und vor allem Bildungs- und Berufserfahrung und sind daher wohl auch mehr in der Lage, realitätsbezogenere Zukunftsvorstellungen zu entwickeln, die dann aber (im Vergleich zu den anderen befragten Gruppen) ausgesprochen pessimistisch und external orientiert sind. Diese Deutung müßte jedoch ebenfalls empirisch abgesichert werden.

Die als sehr wichtig und erwünscht eingestuften Entwicklungen im privaten und beruflichen $\mathrm{Be}$ reich deuten darauf hin, daß sich die von uns 
befragten jungen Frauen auf eine Doppelrolle als Hausfrau und berufstätige Frau einstellen. Erfolg in diesen Rollen scheint ihnen bedeutsamer als den jungen Männern zu sein, wird aber vor allem angesichts antizipierter externer Schwierigkeiten relativ pessimistisch eingeschätzt. Inwieweit die Antizipation solcher erfolgshemmenden Schwierigkeiten gerade durch die Doppelrolle vermittelt wird bzw. dadurch entsteht, daß diese Frauen beruflichen Erfolg anstreben, aber auf ein glückliches Privat- und Familienleben nicht verzichten möchten, müßte erst empirisch geprüft werden - z.B. durch einen Vergleich von Frauen, die primär die eine oder andere Erfuillungsmöglichkeit anstreben, mit Frauen, die hinsichtlich der kognitiv-motivationalen Struktur ihrer Zukunftsorientierung ( $\mathrm{Zu}-$ versicht und Attribuierung) auf die Doppelrolle hin orientiert sind. Vermutlich sind solche $\mathrm{Zu}-$ sammenhänge nicht unabhängig von objektivierbaren sozialen Gegebenheiten, wie Berufschancen sowie normativen und materiellen Anforderungen an die Hausfrauen- und Mutterrolle (vgl. Trommsdorff 1979).

Der Ausbildungsstand und die damit vermittelten Berufschancen sind ein solcher Bedingungsfaktor, der geschlechtsspezifische Differenzen der Zukunftsorientierung beeinflußt. Unsere Ergebnisse deuten im Sinne früherer Befunde zur bildungsspezifischen Differenzierung in der $\mathrm{Zu}$ kunftsorientierung von Jugendlichen (vgl. Lamm et al. 1976) ebenfalls darauf hin, daß die $\mathrm{Zu}$ kunftsorientierung unterer Bildungsgruppen unabhängig von Geschlechtsdifferenzen - weniger differenziert ist. Im Vergleich zu Jugendlichen, die sich noch am Anfang ihrer Ausbildung befinden, haben die Berufsschüler den Vorteil, finanziell eher selbständig zu sein, obwohl sie sich noch in der Ausbildung befinden und ihr Einkommen gering ist. Diese dennoch vermutlich relativ größere Selbständigkeit trägt dazu bei, daß sie zuversichtlicher in die Zukunft blicken und überzeugter von eigenen Handlungserfolgen sind. Diese Deutung läßt sich durch Befunde einer Längsschnittstudie stützen, in der wir bei Jugendlichen, die inzwischen berufstätig geworden waren, eine zunehmende internale Attribuierung feststellten, die insgesamt größer als bei Gymnasiasten war (vgl. Trommsdorff et al. 1979).

Den Berufsschülern bedeutet die materielle Sicherheit mehr als den Gymnasiasten und Stu- denten; dies hängt wohl mit ihrem Ausbildungsziel zusammen, zunächst einmal eine sichere Grundlage für eine stabile berufliche Position zu gewinnen, durch die weitere Wünsche dann eher erfüllbar werden.

Für Gymnasiasten und Studenten dagegen ist bei der jetzigen Arbeitsmarktlage keineswegs eine sichere Koppelung ihrer gegenwärtigen Ausbildung mit einem zukünftigen, auch in materieller Hinsicht befriedigenden Beruf gegeben, so daß die differenzierte Strukturierung von Voraussetzungen bzw. Hindernissen für Erfolg sowie die größere externale Attribuierung und Unsicherheit dieser Jugendlichen durchaus ihrer Lage angemessen und subjektiv realistisch erscheint.

Die hier gefundenen Ergebnisse und ihre teilweise post-hoc Interpretation sind wegen der Stichprobenwahl und -größe nicht einfach zu generalisieren, liefern jedoch Anhaltspunkte für theoretisch bedeutsame Fragestellungen und differenziertere Hypothesen, die in weiteren Untersuchungen empirisch geprüft werden müssen.

Insgesamt machen unsere Ergebnisse deutlich, daß Zukunftsorientierung nicht als einheitliches Konstrukt aufgefaßt, sondern unter Berücksichtigung der subjektiven Erfahrung und Handlungschancen des Individuums hinsichtlich thematischer und kognitiv-motivationaler Komponenten spezifiziert werden sollte.

Als nächstes stellt sich die Frage, inwieweit eher die biologische Geschlechtsrolle oder die subjektive Geschlechtsrolleneinstellung als Prädiktor geeignet ist, um Zukunftsvorstellungen und darauf bezogenes Planen und Handeln vorherzusagen.

Diese und die sich anschließenden Fragen zu Bedingungen sich wandelnder Geschlechtsrollen und Zukunftsvorstellungen sowie daraus sich ergebender Probleme für den Jugendlichen müssen in weiteren Untersuchungen geklärt werden.

Geschlechtsspezifische Differenzen in der Ausprägung solcher Komponenten der Zukunftsorientierung, wie wir sie hier gefunden haben, weisen darauf hin, daß sich traditionelle Geschlechtsrollen teilweise ändern. Dadurch entstehen aber neue Probleme für Frauen, die weiterhin im Rahmen traditioneller Geschlechtsrollenorientierung verarbeitet werden. Die sub- 
jektive Deutung der Zukunft beinhaltet für die Frauen ein höheres Maß an Unsicherheit, Pessimismus und Umweltabhängigkeit als für Männer, insbesondere für Frauen mit schlechterem Ausbildungsniveau.

$\mathrm{Zu}$ fragen wäre, ob diese Art der Antizipation und Bewertung der Zukunft als Element einer subjektiven Handlungstheorie vielleicht eine sich selbst erfüllende Vorhersagefunktion in der Weise hat, daß Pessimismus und externale Attribuierung der Frauen zu Resignation und früherem Verzicht auf das Anstreben erwünschter Entwicklungen fuihren.

\section{Literatur:}

Atkinson, J.W./Raynor, J.O. (Hrsg.), 1974: Motivation and achievement. New York: Wiley.

Crandall, V.C., 1969: Sex differences in expectancy of intellectual and academic reinforcement. In: C.P. Smith (Hrsg.), Achievernent-related motives in children. New York: Russell Sage.

Deaux, K./White, L./Farris, E., 1975: Skill versus luck: Field and laboratory studies of male and female preferences. Journal of Personality and Social Psychology 32: $629-636$.

Feather, N.T., 1969: Attribution of responsibility and valence of success and failure in relation to initial confidence and task performance. Journal of Personality and Social Psychology 13: 129-144.

Füchsle, T./Trommsdorff, G./Burger, C., 1980: Entwicklung eines Meßinstrumentes zur Erfassung der Zukunftsorientierung. Diagnostica 26: 186-197.

Hays, W.L., 1969: Statistics. New York: Holt, Rinehart \& Winston.

Hetherington, E.M. (Hrsg.), 1976: Review of child development research, Vol. 5. Chicago: University of Chicago Press.

Hochreich, D., 1975: Sex-role stereotypes for internalexternal control and interpersonal trust. Journal of Consulting and Clinical Psychology 43: 273.

Horner, M.S., 1972: The motive to avoid success and changing aspirations of college women. In: J.M. Bardwick (Hrsg.), Readings on the psychology of women. New York: Harper \& Row.

Klineberg, S.L., 1968: Future time perspective and the preference for delayed reward. Journal of Personality and Social Psychology 8: 253-257.
Kreutz, H., 1975: Die zeitliche Dimension von Sozialisationsumwelten, Schulbildung, Zukunftsbezug, Selbsteinschätzung und soziale Anpassung von Jugendlichen aus verschiedenen sozialen Schichten. In: H. Walter (Hrsg.), Sozialisationsforschung, Band 3: Sozialökologie. Stuttgart/Bad Cannstadt: Frommann-Holzboog.

Lamm, H./Schmidt, R.W./Trommsdorff, G., 1976: Sex und social class as determinants of future orientation (time perspective) in adolescents. Journal of Personality and Social Psychology 34: 317-326.

Lehr, U., 1972: Das Problem der Sozialisation geschlechtsspezifischer Verhaltensweisen. In: C.F. Graumann (Hrsg.), Handbuch der Psychologie, Band 7, 2. Halbband: Sozialpsychologie. Göttingen: Hogrefe.

Lewin, K., 1948: Time perspective and morale. In: K. Lewin (Hrsg.), Resolving social conflicts. New York: Harper.

Maccoby, E.E./Jacklin, C.N., 1974: The psychology of sex differences. Stanford, California: Stanford University Press.

Nuttin, J./Grommen, R., 1975: Zukunftsperspektive bei Erwachsenen und älteren Menschen aus drei sozioökonomischen Gruppen. In: U. Lehr/F. E. Weinert (Hrsg.), Entwicklung und Persönlichkeit. Stuttgart: Kohlhammer.

Reitz, G., 1974: Die Rolle der Frau und die Lebensplanung der Mädchen: Analysen und Untersuchungen. München: Juventa.

Rosenmayr, L./Kreutz, H., 1973: Rollenerwartungen der weiblichen Jugend. Wien: Österreichischer Bundesverlag.

Sullerot, E. (Hrsg.), 1979: Die Wirklichkeit der Frau. München: Steinhausen.

Tresemer, D., 1977: Fear of success. New York: Plenum.

Trommsdorff, G., 1979: Gesellschaftliche Probleme der Zukunftsorientierung von Jugendlichen. Politik und Kultur 6: 57-66.

Trommsdorff, G./Lamm, H., 1975: An analysis of future orientation and some of its social determinants. S. 343-361 in: J.T. Fraser/N. Lawrence (Hrsg.), The study of time II. New York/Heidelberg: Springer.

Trommsdorff, G./Lamm, H./Schmidt, R.W., 1979: A longitudinal study of adolescents' future orientation (time perspective). Journal of Youth and Adolescence 8: 131-147.

Weiner, B., 1974: Achievement motivation and attribution theory. Morristown, New Jersey: General Learning Press.

Winer, B.J., 1962: Statistical principles in experimental design. New York: McGraw-Hill. 